Original research

\title{
Physical fitness reference standards for preschool children: The PREFIT project
}

\author{
Cristina Cadenas-Sanchez ${ }^{\mathrm{a}, 1}$, *, Timm Intemann ${ }^{\mathrm{b}, 1}$, Idoia Labayen ${ }^{\mathrm{c}}$, Ana B. Peinado ${ }^{\mathrm{d}}$, Josep Vidal-Conti ${ }^{\mathrm{e}}$, \\ Joaquin Sanchís-Moysi ${ }^{\mathrm{f}}$, Diego Moliner-Urdiales ${ }^{\mathrm{g}}$, Manuel A. Rodriguez Perez ${ }^{\mathrm{h}}$, Jorge Cañete Garcia-Prieto ${ }^{\mathrm{i}}$, \\ Jorge del Rosario Fernández-Santos ${ }^{\mathrm{j}}$, Borja Martinez-Tellez ${ }^{\mathrm{a}}$, Germán Vicente-Rodríguez ${ }^{\mathrm{k}}$, Marie Löf $\mathrm{f}^{\mathrm{l}}$
} Jonatan R. Ruiz ${ }^{\mathrm{a}, 1}$, Francisco B. Ortega ${ }^{\mathrm{a}, 1}$ on behalf of the PREFIT project group ${ }^{2}$

a PROFITH "PROmoting FITness and Health through physical activity” Research Group, Department of Physical Education and Sports, Faculty of Sport Sciences, University of Granada, Spain

${ }^{\mathrm{b}}$ Leibniz Institute for Prevention Research and Epidemiology - BIPS, Germany

c Department of Health Sciences, Public University of Navarra, Spain

d Department of Health and Human Performance, Faculty of Physical Activity and Sport Sciences, Technical University of Madrid, Spain

e Department of Education, University of the Balearic Islands, Spain

${ }^{\mathrm{f}}$ Department of Physical Education and Research Institute of Biomedical and Health Sciences (IUIBS), University of Las Palmas de Gran Canaria, Spain

g Department of Education, University Jaume I, Spain

h SPORT Research Group (CTS-1024), Area of Physical Education and Sport, University of Almeria, Spain

${ }^{i}$ University of Castilla-La Mancha, Health and Social Research Center, Spain

j Department of Physical Education, School of Education, University of Cádiz, Spain

" GENUD "Growth, Exercise, NUtrition and Development" Research Group, University of Zaragoza, Spain

${ }^{1}$ Department of Biosciences and Nutrition at NOVUM, Karolinska Institutet, Sweden

\section{A R T I C L E INFO}

Article history:

Received 23 November 2017

Received in revised form 10 September 2018

Accepted 14 September 2018

Available online $\mathrm{xxx}$

\section{Keywords}

Cardiorespiratory fitness

Muscular strength

Motor skills

Reference values

\section{A B S T R A C T}

\section{Objectives}

Reference values are necessary for classifying children, for health screening, and for early prevention as many non-communicable diseases aggravate during growth and development. While physical fitness reference standards are available in children aged 6 and older, such information is lacking in preschool children. Therefore, the purposes of this study were (1) to provide sex-and age-specific physical fitness reference standards for Spanish preschool children; and (2) to study sex differences across this age period and to characterise fitness performance throughout the preschool period.

Design

Cross-sectional study.

Methods

A total of 3179 preschool children (1678 boys) aged 2.8-6.4 years old from 10 cities geographically distributed across Spain were included in the present study. Physical fitness was measured using the PREFIT battery.

Results

Age- and sex-specific percentiles for the main physical fitness components are provided. Boys performed better than girls in the cardiorespiratory fitness, muscular strength, and speed-agility tests over the whole preschool period studied and for the different percentiles. In contrast, girls performed slightly better than boys in the balance test. Older children had better performance in all fitness tests than their younger counterparts. Conclusions

Our study provides age- and sex-specific physical fitness reference standards in preschool children allowing interpretation of fitness assessment. Sexual dimorphism in fitness tests exists already at preschool age, and these differences become larger with age. These findings will help health, sport, and school professionals to identify preschool children with a high/very low fitness level, to examine changes in fitness over time, and to analyse those changes obtained due to intervention effects.

* Corresponding author.

Email address: cadenas@ugr.es (C. Cadenas-Sanchez)

${ }^{1}$ Equal contribution.

${ }^{2}$ See Supplemental material for a complete list of the PREFIT project members. 


\section{Introduction}

Physical fitness is considered a powerful marker of health in children and adolescents. For instance, low fitness levels have been associated with a higher risk of developing cardiovascular diseases, overweight/obesity, mental disorders, and skeletal problems later in life. ${ }^{1,2}$ In line with this notion, Ortega et al. analysed a sample of over one million Swedish adolescents and observed that those with low muscular strength presented higher risk of mortality later in life. ${ }^{3}$ Likewise, in a recent systematic review, the relationship between fitness and health indicators among children and adolescents was examined (including pre-schoolers aged 5). ${ }^{4}$ Although few studies were found in pre-schoolers $(n=5,3.5 \%)$, the results showed significant associations between cardiorespiratory fitness and health indicators, as in studies performed in children and adolescents. ${ }^{4}$ Briefly, cross-sectional and longitudinal studies showed that preschool children aged 5 with higher fitness levels presented lower adiposity and better attention. ${ }^{4}$ Furthermore, our group recently observed that not only cardiorespiratory fitness but also muscular strength, speed-agility, and balance were associated with total and central body fat in 3-5-year-olds. ${ }^{5}$ For this reason, there is a need to include physical fitness testing in health and educational monitoring systems and to examine the associations between fitness and health-related outcomes in 3-5-year-olds.

Fitness reference data have been reported in children and adolescents ( $>6$ years old) from different countries using standardised measures. ${ }^{6-8}$ However, literature addressing reference data of national or international samples including pre-schoolers (3-5 years old) is rather scarce. In fact, as far as we know, only one study provided reference values for one specific test (standing long jump) in pre-schoolers from one province in the south of Spain (Jaén). ${ }^{9}$ Reference values are necessary for classifying children based on their performance on basic motor abilities, for health screening, and for early prevention of biological risk factors for non-communicable diseases (obesity, diabetes, cardiovascular diseases, etc.). Thus, studies providing reference values in preschool children for all fitness components with harmonised measures are warranted.

Therefore, the main objective of this study was to provide sexand age-specific physical fitness reference standards from a sample of preschool children aged 3-5 years old geographically distributed across Spain. This study also addressed sex-related differences across this age period and characterised fitness performance at preschool ages.

\section{Methods}

This study was conducted under the PREFIT project framework (http://profith.ugr.es/prefit). The main objective of this project was to assess physical fitness and anthropometric characteristics in preschool children from 10 different cities/towns in Spain (i.e. Almería, Cádiz, Castellón de la Plana, Cuenca, Granada, Las Palmas de Gran Canaria, Madrid, Palma de Mallorca, Vitoria-Gasteiz, and Zaragoza). The data collection took place from January 2014 to November 2015. The study protocol was approved by the local Review Committee for Research Involving Human Subjects ( $\left.{ }^{\circ} 845\right)$, in accordance with the Declaration of Helsinki 1961 (revision of Edinburgh 2013).

A total of 4338 pre-schoolers and their parents were invited to participate in the PREFIT project. The teaching staff from each school delivered an information sheet and an informed consent to parents and/ or guardians. These included the purpose of the study and brief explanations concerning the applied tests. Finally, 3198 parents agreed to participate in the study (participation rate: $73.7 \%$ ). Among them, 19 children were excluded after the assessments (i.e. they presented a motor or cerebral disease that limited the test performance reported by the school teachers, they cried during most tests, they had a cough and mucus, or they did not understand the instructions of the tests correctly). As a result, a total of 3179 preschool children (4.6 \pm 0.9 years old, 1678 boys, $52.8 \%)$ participated in the PREFIT project (Fig. S1).

We measured children attending first, second, and third grades of preschool centres. In Spain, this mainly includes children aged 3 , 4, and 5 years old. However, these groups included some children younger than $3(n=44,1.4 \%)$ and some older than $5.9(n=112$, $3.5 \%$ ). Tables and Figures report reference values from 3.0 (few participants aged 2 years old) to 6.25 , which corresponds to the first trimester of the 6th year of age. In all the analyses, we merged the data obtained from the youngest and oldest pre-schoolers (i.e., participants aged $<3$ and $\geq 6$ ) with the closest groups for a higher statistical power. Throughout this article, we generally refer to the 3-5-year-old sample, since there are roughly 1000 children who are 3 , 4, and 5 years old and the proportionality out of this range is little $(n=116,4.6 \%)$.

Weight $(\mathrm{kg})$ and height $(\mathrm{cm})$ were assessed without shoes and wearing light clothes using a balance scale (SECA 213, Hamburg, Germany) and a stadiometer (SECA 213, Hamburg, Germany), respectively. Thereafter, we calculated body mass index (BMI) (body mass/body height ${ }^{2}\left[\mathrm{~kg} / \mathrm{m}^{2}\right]$ ).

Physical fitness (i.e. cardiorespiratory fitness, muscular strength, speed-agility, and balance) was assessed with the PREFIT battery. ${ }^{10}$ Feasibility, reliability, maximality, and practical recommendations of these tests have been published elsewhere. ${ }^{11,12}$ Just before the measurements, we told a motivating fairy tale based on Cofito and his adventures on the Lipid Island with the aim to encourage children and make the tests more appealing. More information about this strategy has been published elsewhere. ${ }^{11}$ Cardiorespiratory fitness was assessed with the PREFIT $20 \mathrm{~m}$ shuttle run test that was performed as the last test. Briefly, the test consisted in running back and forth between two lines (20 $\mathrm{m}$ apart) following an audio signal. From the original version proposed by Leger et al. ${ }^{13}$ two modifications were introduced for preschool children ${ }^{12}$ : (1) the test started at $6.5 \mathrm{~km} / \mathrm{h}$ with an increment of $0.5 \mathrm{~km} / \mathrm{h}$ every minute, and (2) one evaluator ran in front of the pre-schoolers and another behind them (e.g. 4-8 pre-schoolers of the same age) in order to help them to maintain the pace. The test finished for each child when they could not reach the line with the audio signal on two consecutive occasions or when they stopped due to fatigue. One of the evaluators was the person responsible for taking out the children of the test when they finished. The test was carried out only once and the resulted laps were registered.

Upper-limb muscular strength was assessed with the handgrip strength test. This test consisted in squeezing as much as possible for 2-3 s. The analogue version of TKK dynamometer (TKK 5001, Grip-A, Takei, Tokyo) was used and the grip span was fixed at $4.0 \mathrm{~cm} .^{14,15}$ The elbow had to be extended without being in contact with anything except for the hand touching the dynamometer. Pre-schoolers performed two non-consecutive attempts with each hand. We chose the best result of each hand and registered the average of both hands in $\mathrm{kg}$.

Lower-limb muscular strength was assessed with the standing long jump test. This test consisted in jumping forward as far as possible, with the feet separated at the shoulders' width, and landing upright. We drew footprints on the floor to guide the pre-schoolers towards the starting line to jump. We recorded the distance between the starting line and the location of the foot closest to the starting line. The children performed the test three times and had time to rest between the attempts. We registered the best of three attempts in $\mathrm{cm}$. 
Speed-agility was assessed with the PREFIT $4 \times 10 \mathrm{~m}$ shuttle run test. In this test, the children had to run four times between two lines (10 $\mathrm{m}$ apart) as fast as possible. Two evaluators stood at each line and the pre-schoolers had to touch the evaluator's hand and return to the starting line as fast as possible. The best of two attempts was manually registered by an experienced evaluator (lowest duration in seconds).

Static balance was assessed with the one-leg stance test. The test consisted in standing on one-leg still and bending the other leg at approximately $90^{\circ}$. The beginning of the test starts when one of the legs is no longer in contact with the floor. The children had to maintain the balance position for as long as they could. In accordance with the original protocol, there were no upper-limb movement restrictions. The test finished when the child could not continue in the required position. The children had one attempt with each leg, and the average time was registered in seconds.

Familiarisation trials and explanations providing examples of how to perform the tests were very important to ensure that the children had understood the process correctly. More information about practical recommendations and how we approached several situations during the assessments can be found in Table S1. The manual of operations, audio of the PREFIT $20 \mathrm{~m}$ shuttle run test, and videos showing how to perform and score the fitness tests are freely available in Spanish and English at: http://profith.ugr.es/recursos-prefit. All the tests were performed by trained evaluators and the protocol was standardised and homogenised across all of the centres involved.

Anthropometric and physical fitness characteristics of the study sample are presented as mean and standard deviation (SD) for the whole sample and stratified by sex and age. We tested differences by sex and age group (3, 4, and 5 years old) with a two-way analysis of variance (ANOVA). The statistical tests were all conducted with a significance level of $\alpha=0.05$. We conducted this statistical analysis using SPSS (v.20, IBM Corporation, New York, USA).

To obtain percentile curves for preschool children, we applied the Generalized Additive Model for Location, Scale and Shape (GAMLSS). ${ }^{16}$ We used the GAMLSS package (version 4.4-0) of the statistical software R (version 3.3.1). GAMLSS is able to model up to four parameters of different distributions: $\mu$ accounts for the location, $\sigma$ for the scale, $v$ for the skewness, and $\tau$ for the kurtosis. The Box-Cox Cole and Green, Box-Cox $t$, and Box-Cox power exponential distribution were fitted to the observed data. Furthermore, the influence of age on the distribution parameters was modelled constantly, linearly, or as a cubic spline function. We assessed the goodness of fit applying the Bayesian information criterion and worm plots. More information about the procedure has been described elsewhere. ${ }^{17}$ Percentile curves for the 1st, 3rd, 5th, 10th, 15th, 20th, 30th, 40th, 50th, 60th, 70th, 80th, 85th, 90th, 95th, 97th, and 99th percentiles were calculated based on the model that showed the best goodness of fit (Table S2 and Supplementary material 2). We provide reference standards with a precision of 0.25 years of age (every trimester) as the main outcome of this article and also every 0.025 years of age as supplementary material 1 (equivalent to 9 days). To test sex differences in fitness across age groups and percentiles 25 th, 50th, and 75 th, we performed a one-way ANOVA including the sex differences as dependent variables and age groups $(3,4$, and 5 years old) as factor. We used these percentiles to test sex differences in fit pre-schoolers (percentile 75th), in averagely fit pre-schoolers (percentile 50th), and in unfit pre-schoolers (percentile 25 th). We had previously calculated and depicted mean differences for every 0.05 years of age (i.e. 9 days) and percentiles.

\section{Results}

Anthropometric and physical fitness characteristics of the study sample (whole sample and separated by sex and age) are shown in Table S3.

Reference standards for the 1st, 3rd, 5th, 10th, 15th, 20th, 30th, 40th, 50th, 60th, 70th, 80th, 85th, 90th, 95th, 97th, and 99th percentiles and for every 0.025 years of age (i.e. 9 days) are provided in supplementary material 1 (as Excel file). A summary of these reference standards (i.e. percentiles: 10th, 20th, 30th, 40th, 50th, 60th, 70th, 80th, 90th, 99th) for each trimester (i.e. 0.25 years of age, 3 months) is provided in Tables 1 and 2. Fig. 1 depicts sex- and age-specific fitness reference data according to the 1st, 5th, 15th, 25th, 50th, 75th, 85th, 95th, and 99th percentiles. We found higher values in boys compared to girls in the entire fitness tests battery except for the one-leg stance test, where girls showed better performance in all analysed percentiles. Also, along the analysed percentiles, the performance improved with age. We found larger differences between P95 and P99 in older preschool children than in their younger counterparts in the PREFIT $20 \mathrm{~m}$ shuttle run test (for girls), the standing long jump test (for girls), and the one-leg stance test (for boys and girls) (Fig. 1). In the $4 \times 10 \mathrm{~m}$ shuttle run test, younger children showed larger differences (for boys and girls) not only between P95 and P99 but also for P1 and P5 percentiles (Fig. 1). Fig. S2 shows sex differences across the preschool age and percentiles 25 th, 50 th, and 75 th. Table S4 shows mean differences between boys and girls in all examined age groups. We found significant differences in most of the fitness components and percentiles studied (all $\mathrm{p} \leq 0.001$ ), except in the standing long jump (for P75 between 3 and 5 years old) and speed-agility (P50 and P75, between 4 and 5 years old and between 3 and 5 years old, respectively) tests, where no differences between boys and girls were observed (all $\mathrm{p}>0.05)$.

\section{Discussion}

The present study provides, for the first time, reference standards for the main fitness components (i.e. cardiorespiratory fitness, muscular strength, speed-agility, and balance) by sex and age (for every 0.025 year of age increment, i.e. 9 days) in preschool children. Boys performed better than girls in cardiorespiratory fitness, muscular strength, and speed-agility tests, whereas girls performed slightly better in balance tests. Older preschool children performed better in all fitness tests than younger pre-schoolers. Furthermore, we observed sex-related differences in fitness across all preschool ages and percentiles, being greater in older children.

In regard to sex differences, from as early as preschool age, boys showed higher levels of physical fitness (i.e. cardiorespiratory fitness, muscular strength, and speed-agility) than girls, as it had previously been reported in older children and adolescents. ${ }^{7,18,19}$ However, in relation to balance, girls performed slightly better in the one-leg stance test compared to boys. These differences might be explained by differential sex development and growth. Our results are in accordance with recent studies that analysed motor proficiency at early childhood. Such studies conclude that boys perform better than girls in activities such as catching, throwing, or standing long jump. ${ }^{20,21}$ Conversely, girls perform better in activities involving balance or flexibility. ${ }^{21,22}$ These differences have previously been addressed in adolescents, but not in preschool children. ${ }^{23,24}$ Studies showed that boys have higher levels of cardiorespiratory fitness because they are more physically active $e^{23}$ and have lower levels of fat mass. ${ }^{24-26}$ In the same way, based on the previous literature, fat-free mass is higher in boys, ${ }^{27}$ which allows them to perform better in muscular strength tests. $^{24}$ In regard to speed-agility, other authors showed that the per- 
Table 1

Reference standards of cardiorespiratory fitness and muscular strength tests calculated with Generalized Additive Model for Location, Scale and Shape (GAMLSS) in preschool children.

\begin{tabular}{|c|c|c|c|c|c|c|c|c|c|c|c|c|c|c|c|c|c|c|c|c|c|}
\hline Age (years) & Boys & & & & & & & & & & Age (years) & Girls & & & & & & & & & \\
\hline & P10 & P20 & P30 & P40 & P50 & P60 & P70 & P80 & $\mathrm{P} 90$ & P99 & & P10 & P20 & P30 & P40 & P50 & P60 & P70 & P80 & P90 & P99 \\
\hline \multicolumn{22}{|c|}{ Cardiorespiratory fitness: PREFIT $20 \mathrm{~m}$ shuttle run test (laps) } \\
\hline $3.00-3.24$ & 4 & 5 & 6 & 7 & 8 & 10 & 12 & 14 & 18 & 32 & $3.00-3.24$ & 4 & 5 & 6 & 7 & 8 & 9 & 10 & 11 & 14 & 21 \\
\hline $3.25-3.49$ & 5 & 6 & 7 & 9 & 10 & 12 & 14 & 17 & 21 & 34 & $3.25-3.49$ & 5 & 6 & 7 & 8 & 9 & 10 & 12 & 14 & 17 & 25 \\
\hline $3.50-3.74$ & 6 & 7 & 9 & 11 & 12 & 14 & 17 & 20 & 24 & 37 & $3.50-3.74$ & 6 & 7 & 8 & 10 & 11 & 12 & 14 & 16 & 19 & 30 \\
\hline $3.75-3.99$ & 7 & 9 & 11 & 12 & 14 & 17 & 19 & 22 & 27 & 40 & $3.75-3.99$ & 6 & 8 & 10 & 11 & 12 & 14 & 16 & 18 & 22 & 34 \\
\hline $4.00-4.24$ & 8 & 10 & 12 & 14 & 16 & 19 & 21 & 25 & 30 & 43 & $4.00-4.24$ & 7 & 9 & 11 & 12 & 14 & 16 & 18 & 21 & 25 & 39 \\
\hline $4.25-4.49$ & 9 & 12 & 14 & 16 & 18 & 21 & 24 & 27 & 32 & 46 & $4.25-4.49$ & 8 & 10 & 12 & 14 & 16 & 18 & 20 & 23 & 28 & 43 \\
\hline $4.50-4.74$ & 10 & 13 & 15 & 18 & 20 & 23 & 26 & 30 & 35 & 49 & $4.50-4.74$ & 9 & 11 & 13 & 15 & 17 & 19 & 22 & 25 & 31 & 47 \\
\hline $4.75-4.99$ & 11 & 14 & 17 & 20 & 22 & 25 & 29 & 32 & 38 & 52 & $4.75-4.99$ & 10 & 12 & 14 & 16 & 19 & 21 & 24 & 27 & 33 & 51 \\
\hline $5.00-5.24$ & 12 & 16 & 19 & 21 & 24 & 27 & 31 & 35 & 41 & 56 & $5.00-5.24$ & 10 & 13 & 15 & 18 & 20 & 23 & 26 & 30 & 36 & 55 \\
\hline $5.25-5.49$ & 13 & 17 & 20 & 23 & 26 & 30 & 33 & 38 & 44 & 59 & $5.25-5.49$ & 11 & 14 & 16 & 19 & 21 & 24 & 27 & 32 & 38 & 59 \\
\hline $5.50-5.74$ & 14 & 18 & 22 & 25 & 28 & 32 & 36 & 40 & 47 & 64 & $5.50-5.74$ & 12 & 15 & 18 & 20 & 23 & 26 & 29 & 33 & 41 & 63 \\
\hline $5.75-5.99$ & 16 & 20 & 23 & 27 & 30 & 34 & 38 & 43 & 50 & 68 & $5.75-5.99$ & 12 & 16 & 19 & 21 & 24 & 27 & 31 & 35 & 43 & 66 \\
\hline $6.00-6.25$ & 17 & 21 & 25 & 29 & 32 & 36 & 41 & 46 & 53 & 72 & $6.00-6.25$ & 13 & 17 & 20 & 22 & 25 & 29 & 32 & 37 & 45 & 70 \\
\hline \multicolumn{22}{|c|}{ Upper-limb muscular strength: handgrip strength test (kg) } \\
\hline $3.00-3.24$ & 2.6 & 3.2 & 3.6 & 4.0 & 4.3 & 4.7 & 5.0 & 5.5 & 6.2 & 8.0 & $3.00-3.24$ & 2.3 & 2.9 & 3.3 & 3.6 & 3.9 & 4.2 & 4.5 & 5.0 & 5.6 & 7.7 \\
\hline $3.25-3.49$ & 3.0 & 3.6 & 4.1 & 4.5 & 4.8 & 5.2 & 5.6 & 6.1 & 6.8 & 8.8 & $3.25-3.49$ & 2.7 & 3.3 & 3.7 & 4.0 & 4.3 & 4.7 & 5.0 & 5.5 & 6.2 & 8.2 \\
\hline $3.50-3.74$ & 3.4 & 4.1 & 4.5 & 4.9 & 5.3 & 5.7 & 6.2 & 6.7 & 7.4 & 9.5 & $3.50-3.74$ & 3.1 & 3.7 & 4.1 & 4.5 & 4.8 & 5.2 & 5.6 & 6.0 & 6.7 & 8.9 \\
\hline $3.75-3.99$ & 3.8 & 4.5 & 5.0 & 5.4 & 5.8 & 6.3 & 6.7 & 7.3 & 8.1 & 10.2 & $3.75-3.99$ & 3.4 & 4.1 & 4.5 & 4.9 & 5.3 & 5.6 & 6.1 & 6.6 & 7.3 & 9.4 \\
\hline $4.00-4.24$ & 4.2 & 4.9 & 5.5 & 5.9 & 6.4 & 6.8 & 7.3 & 7.8 & 8.7 & 10.9 & $4.00-4.24$ & 3.8 & 4.5 & 4.9 & 5.3 & 5.7 & 6.1 & 6.6 & 7.1 & 7.8 & 9.9 \\
\hline $4.25-4.49$ & 4.6 & 5.4 & 5.9 & 6.4 & 6.9 & 7.3 & 7.8 & 8.4 & 9.2 & 11.5 & $4.25-4.49$ & 4.2 & 4.9 & 5.4 & 5.8 & 6.2 & 6.6 & 7.0 & 7.6 & 8.3 & 10.3 \\
\hline $4.50-4.74$ & 5.1 & 5.9 & 6.4 & 6.9 & 7.4 & 7.8 & 8.3 & 9.0 & 9.8 & 12.2 & $4.50-4.74$ & 4.6 & 5.3 & 5.8 & 6.2 & 6.7 & 7.1 & 7.5 & 8.1 & 8.9 & 10.8 \\
\hline $4.75-4.99$ & 5.5 & 6.3 & 6.9 & 7.4 & 7.9 & 8.4 & 8.9 & 9.5 & 10.4 & 12.8 & $4.75-4.99$ & 5.0 & 5.7 & 6.2 & 6.7 & 7.1 & 7.6 & 8.0 & 8.6 & 9.4 & 11.4 \\
\hline $5.00-5.24$ & 6.0 & 6.8 & 7.4 & 7.9 & 8.4 & 8.9 & 9.4 & 10.1 & 11.0 & 13.4 & $5.00-5.24$ & 5.4 & 6.1 & 6.7 & 7.1 & 7.6 & 8.0 & 8.5 & 9.1 & 9.9 & 11.9 \\
\hline $5.25-5.49$ & 6.4 & 7.3 & 7.9 & 8.4 & 8.9 & 9.4 & 9.9 & 10.6 & 11.5 & 14.0 & $5.25-5.49$ & 5.8 & 6.6 & 7.1 & 7.6 & 8.0 & 8.5 & 9.0 & 9.6 & 10.4 & 12.5 \\
\hline $5.50-5.74$ & 6.9 & 7.8 & 8.4 & 8.9 & 9.4 & 9.9 & 10.5 & 11.1 & 12.1 & 14.5 & $5.50-5.74$ & 6.2 & 7.0 & 7.6 & 8.0 & 8.5 & 9.0 & 9.5 & 10.1 & 11.0 & 13.1 \\
\hline $5.75-5.99$ & 7.4 & 8.2 & 8.9 & 9.4 & 9.9 & 10.4 & 11.0 & 11.7 & 12.6 & 15.1 & $5.75-5.99$ & 6.6 & 7.4 & 8.0 & 8.5 & 9.0 & 9.5 & 10.0 & 10.6 & 11.5 & 13.7 \\
\hline $6.00-6.25$ & 7.8 & 8.7 & 9.4 & 9.9 & 10.4 & 11.0 & 11.5 & 12.2 & 13.2 & 15.6 & $6.00-6.25$ & 7.0 & 7.8 & 8.4 & 8.9 & 9.4 & 9.9 & 10.5 & 11.1 & 12.0 & 14.3 \\
\hline \multicolumn{22}{|c|}{ Lower-limb muscular strength: standing long jump $(\mathrm{cm})$} \\
\hline $3.00-3.24$ & 36.4 & 42.0 & 45.8 & 49.0 & 51.9 & 54.7 & 57.8 & 61.4 & 66.6 & 79.4 & $3.00-3.24$ & 30.4 & 35.7 & 39.4 & 42.5 & 45.2 & 48.0 & 51.0 & 54.5 & 59.4 & 71.5 \\
\hline $3.25-3.49$ & 39.8 & 46.0 & 50.2 & 53.6 & 56.7 & 59.8 & 63.2 & 67.2 & 72.9 & 86.9 & $3.25-3.49$ & 33.7 & 39.6 & 43.7 & 47.0 & 50.0 & 53.0 & 56.3 & 60.1 & 65.7 & 79.3 \\
\hline $3.50-3.74$ & 43.3 & 50.0 & 54.6 & 58.3 & 61.7 & 65.0 & 68.6 & 72.9 & 79.1 & 94.6 & $3.50-3.74$ & 36.9 & 43.5 & 47.9 & 51.5 & 54.8 & 58.0 & 61.5 & 65.7 & 71.8 & 87.1 \\
\hline $3.75-3.99$ & 46.8 & 54.1 & 59.0 & 63.0 & 66.5 & 70.1 & 74.0 & 78.7 & 85.4 & 102.2 & $3.75-3.99$ & 40.1 & 47.2 & 52.0 & 55.9 & 59.3 & 62.7 & 66.4 & 71.0 & 77.6 & 94.5 \\
\hline $4.00-4.24$ & 50.2 & 58.0 & 63.2 & 67.5 & 71.3 & 75.0 & 79.2 & 84.2 & 91.4 & 109.6 & $4.00-4.24$ & 43.1 & 50.8 & 55.9 & 60.0 & 63.6 & 67.2 & 71.1 & 76.0 & 83.2 & 101.8 \\
\hline $4.25-4.49$ & 53.2 & 61.5 & 67.1 & 71.5 & 75.5 & 79.5 & 83.9 & 89.2 & 96.8 & 116.3 & $4.25-4.49$ & 46.1 & 54.3 & 59.7 & 64.0 & 67.7 & 71.4 & 75.6 & 80.8 & 88.5 & 108.8 \\
\hline $4.50-4.74$ & 56.0 & 64.7 & 70.5 & 75.2 & 79.3 & 83.5 & 88.0 & 93.6 & 101.7 & 122.3 & $4.50-4.74$ & 48.9 & 57.7 & 63.4 & 67.9 & 71.7 & 75.6 & 79.9 & 85.4 & 93.6 & 115.6 \\
\hline $4.75-4.99$ & 58.4 & 67.5 & 73.6 & 78.4 & 82.7 & 87.0 & 91.7 & 97.5 & 105.9 & 127.7 & $4.75-4.99$ & 51.7 & 61.0 & 66.9 & 71.6 & 75.5 & 79.5 & 84.0 & 89.7 & 98.4 & 122.1 \\
\hline $5.00-5.24$ & 60.6 & 70.0 & 76.3 & 81.3 & 85.7 & 90.1 & 95.0 & 101.0 & 109.7 & 132.5 & $5.00-5.24$ & 54.2 & 64.0 & 70.2 & 75.0 & 79.0 & 83.0 & 87.7 & 93.6 & 102.8 & 128.1 \\
\hline $5.25-5.49$ & 62.6 & 72.4 & 78.9 & 84.0 & 88.5 & 93.0 & 98.0 & 104.2 & 113.3 & 137.0 & $5.25-5.49$ & 56.6 & 66.8 & 73.2 & 78.0 & 82.1 & 86.2 & 91.0 & 97.1 & 106.7 & 133.7 \\
\hline $5.50-5.74$ & 64.5 & 74.6 & 81.3 & 86.5 & 91.1 & 95.7 & 100.8 & 107.2 & 116.6 & 141.3 & $5.50-5.74$ & 58.7 & 69.3 & 75.8 & 80.8 & 84.9 & 89.0 & 93.8 & 100.1 & 110.1 & 138.6 \\
\hline $5.75-5.99$ & 66.4 & 76.8 & 83.6 & 89.0 & 93.6 & 98.3 & 103.5 & 110.0 & 119.7 & 145.3 & $5.75-5.99$ & 60.6 & 71.5 & 78.2 & 83.2 & 87.3 & 91.4 & 96.3 & 102.8 & 113.0 & 143.1 \\
\hline $6.00-6.25$ & 68.2 & 78.9 & 85.8 & 91.3 & 96.1 & 100.8 & 106.1 & 112.8 & 122.8 & 149.3 & $6.00-6.25$ & 62.4 & 73.6 & 80.4 & 85.5 & 89.5 & 93.6 & 98.6 & 105.2 & 115.7 & 147.3 \\
\hline
\end{tabular}

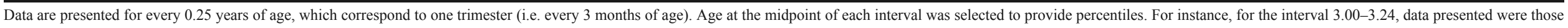
corresponding to an exact age of 3.125 years old.

P10: 10th percentile; other percentiles are abbreviated accordingly. 
Table 2

Reference standards of speed-agility and balance tests calculated with Generalized Additive Model for Location, Scale and Shape (GAMLSS) in preschool children.

\begin{tabular}{|c|c|c|c|c|c|c|c|c|c|c|c|c|c|c|c|c|c|c|c|c|c|}
\hline Age (years) & Boys & & & & & & & & & & Age (years) & Girls & & & & & & & & & \\
\hline & P10 & P20 & P30 & P40 & P50 & P60 & P70 & P80 & P90 & P99 & & P10 & P20 & P30 & P40 & P50 & P60 & P70 & P80 & P90 & P99 \\
\hline \multicolumn{22}{|c|}{ Speed-agility: $4 \times 10 \mathrm{~m}$ shuttle run test (s) } \\
\hline $3.00-3.24$ & 17.2 & 18.0 & 18.6 & 19.1 & 19.7 & 20.2 & 20.9 & 21.7 & 23.0 & 27.2 & $3.00-3.24$ & 17.8 & 18.8 & 19.4 & 20.0 & 20.5 & 21.0 & 21.7 & 22.5 & 24.0 & 29.0 \\
\hline $3.25-3.49$ & 16.6 & 17.4 & 18.0 & 18.5 & 19.0 & 19.5 & 20.1 & 20.8 & 22.0 & 25.9 & $3.25-3.49$ & 17.3 & 18.2 & 18.8 & 19.3 & 19.7 & 20.2 & 20.8 & 21.6 & 22.8 & 27.2 \\
\hline $3.50-3.74$ & 16.1 & 16.8 & 17.3 & 17.8 & 18.3 & 18.8 & 19.3 & 20.0 & 21.1 & 24.6 & $3.50-3.74$ & 16.8 & 17.6 & 18.1 & 18.6 & 19.0 & 19.4 & 19.9 & 20.6 & 21.8 & 25.6 \\
\hline $3.75-3.99$ & 15.6 & 16.3 & 16.8 & 17.2 & 17.6 & 18.1 & 18.6 & 19.2 & 20.2 & 23.4 & $3.75-3.99$ & 16.3 & 17.0 & 17.5 & 17.9 & 18.3 & 18.6 & 19.1 & 19.7 & 20.8 & 24.1 \\
\hline $4.00-4.24$ & 15.1 & 15.7 & 16.2 & 16.6 & 17.0 & 17.4 & 17.9 & 18.5 & 19.4 & 22.3 & $4.00-4.24$ & 15.7 & 16.4 & 16.8 & 17.2 & 17.6 & 17.9 & 18.4 & 19.0 & 19.9 & 22.8 \\
\hline $4.25-4.49$ & 14.7 & 15.3 & 15.7 & 16.1 & 16.5 & 16.9 & 17.3 & 17.8 & 18.7 & 21.4 & $4.25-4.49$ & 15.2 & 15.8 & 16.3 & 16.6 & 17.0 & 17.4 & 17.8 & 18.3 & 19.2 & 21.7 \\
\hline $4.50-4.74$ & 14.3 & 14.9 & 15.3 & 15.7 & 16.0 & 16.4 & 16.8 & 17.3 & 18.1 & 20.6 & $4.50-4.74$ & 14.8 & 15.3 & 15.8 & 16.1 & 16.5 & 16.9 & 17.3 & 17.8 & 18.6 & 20.9 \\
\hline $4.75-4.99$ & 14.0 & 14.5 & 14.9 & 15.3 & 15.6 & 15.9 & 16.3 & 16.8 & 17.5 & 19.9 & $4.75-4.99$ & 14.4 & 15.0 & 15.4 & 15.7 & 16.1 & 16.5 & 16.9 & 17.4 & 18.2 & 20.3 \\
\hline $5.00-5.24$ & 13.7 & 14.2 & 14.6 & 14.9 & 15.2 & 15.5 & 15.9 & 16.4 & 17.1 & 19.4 & $5.00-5.24$ & 14.1 & 14.7 & 15.1 & 15.4 & 15.8 & 16.1 & 16.6 & 17.1 & 17.8 & 19.9 \\
\hline $5.25-5.49$ & 13.4 & 13.9 & 14.3 & 14.6 & 14.9 & 15.2 & 15.6 & 16.0 & 16.7 & 18.9 & $5.25-5.49$ & 13.9 & 14.4 & 14.8 & 15.1 & 15.5 & 15.8 & 16.2 & 16.7 & 17.5 & 19.5 \\
\hline $5.50-5.74$ & 13.1 & 13.6 & 14.0 & 14.3 & 14.6 & 14.9 & 15.3 & 15.7 & 16.4 & 18.5 & $5.50-5.74$ & 13.6 & 14.1 & 14.5 & 14.9 & 15.2 & 15.5 & 15.9 & 16.4 & 17.1 & 19.1 \\
\hline $5.75-5.99$ & 12.9 & 13.4 & 13.7 & 14.0 & 14.3 & 14.6 & 15.0 & 15.4 & 16.1 & 18.1 & $5.75-5.99$ & 13.3 & 13.8 & 14.2 & 14.6 & 14.9 & 15.2 & 15.6 & 16.1 & 16.8 & 18.7 \\
\hline $6.00-6.25$ & 12.7 & 13.2 & 13.5 & 13.8 & 14.1 & 14.4 & 14.7 & 15.1 & 15.8 & 17.8 & $6.00-6.25$ & 13.1 & 13.5 & 13.9 & 14.2 & 14.6 & 14.9 & 15.3 & 15.8 & 16.4 & 18.2 \\
\hline \multicolumn{22}{|c|}{ Balance: one-leg stance test (seconds) } \\
\hline $3.00-3.24$ & 1.1 & 1.5 & 1.8 & 2.2 & 2.7 & 3.2 & 3.9 & 4.9 & 6.8 & 16.2 & $3.00-3.24$ & 1.0 & 1.5 & 1.8 & 2.3 & 2.7 & 3.3 & 4.0 & 5.1 & 7.0 & 15.1 \\
\hline $3.25-3.49$ & 1.4 & 1.9 & 2.3 & 2.8 & 3.3 & 4.0 & 4.9 & 6.1 & 8.6 & 20.3 & $3.25-3.49$ & 1.4 & 2.0 & 2.6 & 3.1 & 3.8 & 4.6 & 5.6 & 7.0 & 9.7 & 21.0 \\
\hline $3.50-3.74$ & 1.7 & 2.3 & 2.8 & 3.4 & 4.1 & 4.9 & 6.0 & 7.5 & 10.5 & 25.0 & $3.50-3.74$ & 1.9 & 2.6 & 3.4 & 4.1 & 5.0 & 6.0 & 7.3 & 9.2 & 12.7 & 27.5 \\
\hline $3.75-3.99$ & 2.1 & 2.8 & 3.5 & 4.2 & 5.0 & 6.0 & 7.3 & 9.2 & 12.9 & 30.6 & $3.75-3.99$ & 2.4 & 3.3 & 4.2 & 5.2 & 6.3 & 7.5 & 9.2 & 11.6 & 16.0 & 34.7 \\
\hline $4.00-4.24$ & 2.5 & 3.4 & 4.2 & 5.1 & 6.1 & 7.3 & 8.9 & 11.2 & 15.7 & 37.2 & $4.00-4.24$ & 2.9 & 4.1 & 5.2 & 6.4 & 7.7 & 9.3 & 11.3 & 14.3 & 19.7 & 42.6 \\
\hline $4.25-4.49$ & 3.0 & 4.1 & 5.1 & 6.2 & 7.4 & 8.8 & 10.7 & 13.5 & 18.9 & 44.8 & $4.25-4.49$ & 3.5 & 4.9 & 6.2 & 7.6 & 9.2 & 11.1 & 13.5 & 17.1 & 23.6 & 51.0 \\
\hline $4.50-4.74$ & 3.6 & 4.9 & 6.1 & 7.3 & 8.7 & 10.5 & 12.7 & 16.0 & 22.4 & 53.2 & $4.50-4.74$ & 4.1 & 5.7 & 7.3 & 9.0 & 10.8 & 13.0 & 15.9 & 20.1 & 27.7 & 59.9 \\
\hline $4.75-4.99$ & 4.2 & 5.7 & 7.1 & 8.6 & 10.2 & 12.2 & 14.9 & 18.8 & 26.2 & 62.3 & $4.75-4.99$ & 4.7 & 6.6 & 8.4 & 10.3 & 12.5 & 15.1 & 18.4 & 23.2 & 32.0 & 69.2 \\
\hline $5.00-5.24$ & 4.9 & 6.6 & 8.2 & 9.9 & 11.8 & 14.2 & 17.2 & 21.7 & 30.3 & 72.0 & $5.00-5.24$ & 5.4 & 7.6 & 9.6 & 11.8 & 14.2 & 17.1 & 20.9 & 26.4 & 36.4 & 78.8 \\
\hline $5.25-5.49$ & 5.6 & 7.5 & 9.4 & 11.3 & 13.5 & 16.1 & 19.6 & 24.7 & 34.5 & 82.0 & $5.25-5.49$ & 6.0 & 8.5 & 10.8 & 13.2 & 16.0 & 19.3 & 23.5 & 29.7 & 40.9 & 88.5 \\
\hline $5.50-5.74$ & 6.2 & 8.4 & 10.5 & 12.7 & 15.1 & 18.1 & 22.0 & 27.8 & 38.8 & 92.2 & $5.50-5.74$ & 6.7 & 9.4 & 12.0 & 14.7 & 17.7 & 21.4 & 26.1 & 32.9 & 45.4 & 98.3 \\
\hline $5.75-5.99$ & 6.9 & 9.4 & 11.7 & 14.1 & 16.8 & 20.1 & 24.5 & 30.9 & 43.1 & 102.4 & $5.75-5.99$ & 7.4 & 10.4 & 13.2 & 16.1 & 19.5 & 23.5 & 28.7 & 36.2 & 50.0 & 108.1 \\
\hline $6.00-6.25$ & 7.6 & 10.3 & 12.9 & 15.5 & 18.5 & 22.2 & 26.9 & 34.0 & 47.5 & 112.7 & $6.00-6.25$ & 8.0 & 11.3 & 14.4 & 17.6 & 21.2 & 25.6 & 31.3 & 39.5 & 54.4 & 117.8 \\
\hline
\end{tabular}

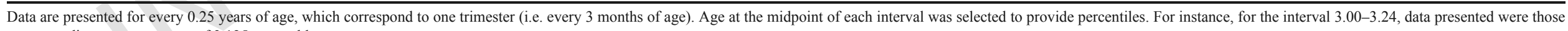
corresponding to an exact age of 3.125 years old

P10: 10th percentile; other percentiles are abbreviated accordingly. 

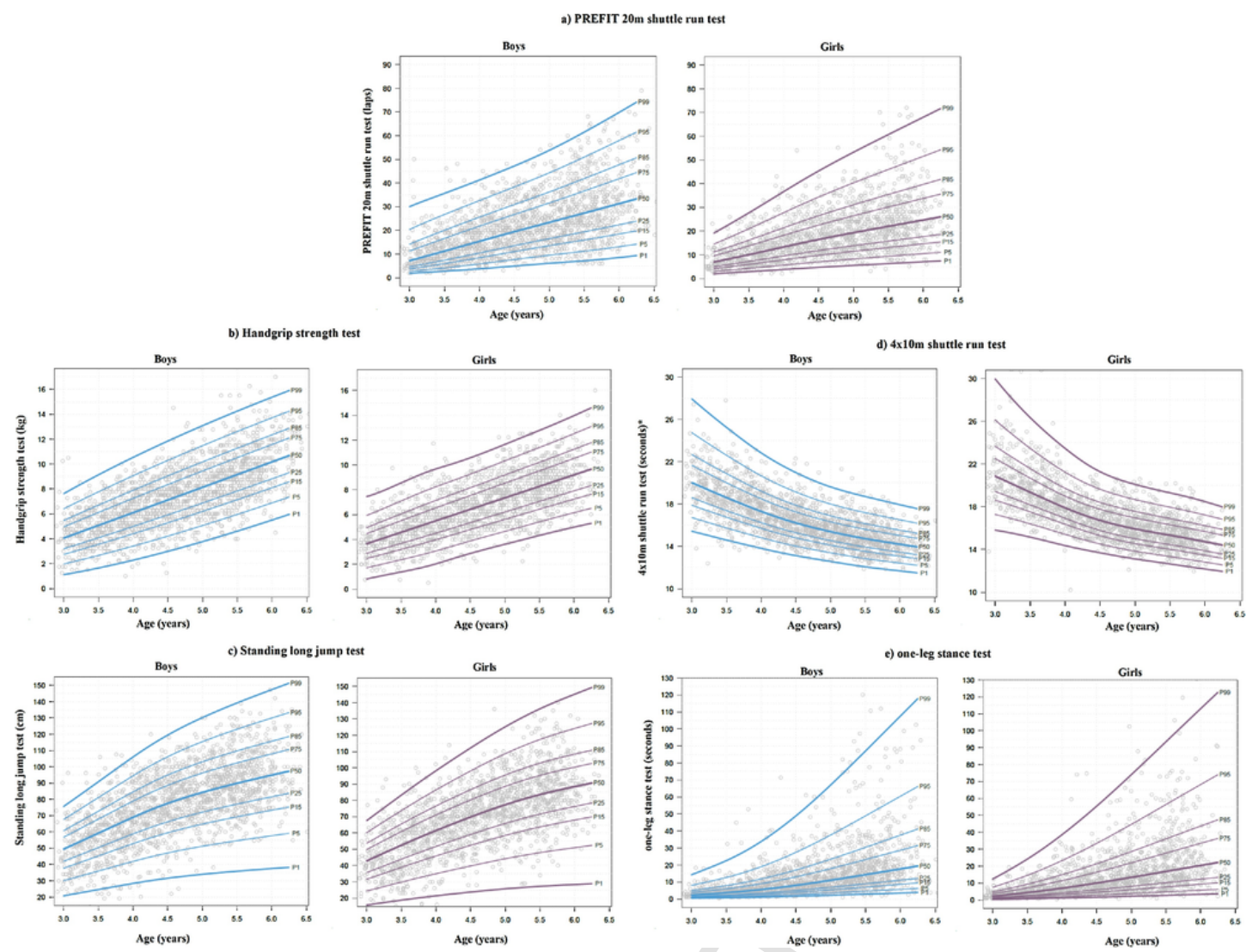

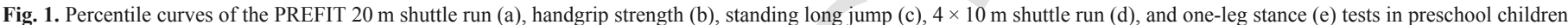

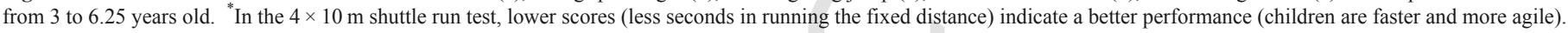

formance seems to be influenced by genetics (neuromuscular components, muscle fibre quality, degree of gene transfer, etc.), and thus, there is no explanation for the sex-related differences. ${ }^{24,25}$ Likewise, it is possible that height could explain the sex-differences observed in balance, since taller children (in average boys are taller than girls already at these ages) would have a higher postural instability and thus worse balance as a result of a higher location of the centre of body mass. ${ }^{28}$ Our results cannot support these assumptions since we have not measured fat-mass, fat-free mass, or physical activity. However, although these differences have been observed in adolescents, there is no reason to believe that similar physiological differences could also be found already in children aged 3-5 years old.

Preschool children are in a period of continuous motor, physiological, and psychological developing changes. ${ }^{29} \mathrm{We}$ observed that older children performed better in all fitness tests since their motor development and fitness were higher (i.e. better aerobic capacity, muscular strength, coordination, agility, etc.) compared to their younger counterparts. We observed similar patterns in children and adolescents in most of the study test, ${ }^{7,18,30}$ except in those assessing flexibility. Flexibility tests showed that, overall, the performance improved with age in girls ${ }^{7,18,30}$ and remained stable or became worse in boys. ${ }^{30}$ Other factors that could explain these differences are motivation, concentration, degree of the motor skills, physical activity, or body composition (fat mass and fat-free mass). ${ }^{29}$

To the best of our knowledge, our study is the first to provide age- and sex-specific reference standards of a complete set of physical fitness components (i.e., cardiorespiratory fitness, muscular strength, speed-agility, and balance) in preschool children. A strength is that the applied tests were selected based on a systematic review in preschool children, ${ }^{10}$ together with the existing evidence in older children and adolescents. ${ }^{1}$ Previous studies ${ }^{7,8,18}$ showed reference data for cardiorespiratory fitness, muscular strength, speed-agility, and flexibility in children and adolescents ( $>6$ years old) using evidence-based fitness tests batteries (e.g. ALPHA). Nevertheless, our data are not fully comparable due to the differences in age groups and tests applied.

In regard to cardiorespiratory fitness, we observed that the differences of the 50th percentiles (P50) between both sexes increased with age (Fig. 1). Although we conducted different tests (original $20 \mathrm{~m}$ shuttle run vs. PREFIT $20 \mathrm{~m}$ shuttle run) and analysed different age groups, other studies in children and adolescents showed a similar trend in P50 (e.g. sex-differences in 18-year-old adolescents: +38 laps for boys). ${ }^{7,19}$ Higher age-related differences by sex observed in adolescents compared to children or even preschool children might be explained by more pronounced physiological changes (due to the pubertal development) that occur as age increases. ${ }^{31}$

Upper-limb muscular strength, assessed by the handgrip strength test, showed sex-differences in P50 of approximately $1 \mathrm{~kg}$ as age increased 0.5 years. Despite differences between studies and devices (analogue vs. digital dynamometer), our results are in accordance with the results of De Miguel-Etayo et al., ${ }^{7}$ who observed the same sex-differences in children aged 6-9 years old. Given that the methodology of the test is the same and the inter-instrument reliability is high (mean difference, digital minus analogue dynamometer $=-0.35 \mathrm{~kg}$ ), 15 our results are comparable with groups of 6-year-olds. The preschool children from the PREFIT project were stronger than the IDEFICS children (mean differences: 1.28 and $1.29 \mathrm{~kg}$ for boys and girls, respectively). Roriz De Oliveira et al. ${ }^{30}$ also provided reference values for Portuguese children aged 6-10 (age-range 1 year), showing similar sex-differences (nearly $1 \mathrm{~kg}$ ). They also reported up- 
per-limb muscular strength reference standard in P50, which is slightly lower compared to our results. The differences between studies might be due to the limitations of the digital dynamometer, since the range of measurement is from $5 \mathrm{~kg}$ to $100 \mathrm{~kg}$ and the fact that 6 -year-olds had several attempts below this range (i.e., $0 \mathrm{~kg}$ ). ${ }^{15} \mathrm{An}$ other remarkable difference between the IDEFICS and the PREFIT studies is that overweight and obese children were not included in the calculation of reference standards in IDEFICS. Indeed, children and adolescents with higher BMI performed better in tests assessing absolute strength (also called non-weight bearing test) ${ }^{24}$ compared to those with lower BMI.

Regarding lower-limb muscular strength, we observed that the differences in P50 between both sexes ranged from 6 to $8 \mathrm{~cm}$ as age increased 0.5 years. Latorre-Roman et al. ${ }^{9}$ provided reference data in standing long jump in a group of 3-6-year-olds from Jaén (a region from the south of Spain). It can be observed that P50 of the PREFIT reference data of this study was slightly higher in boys and girls and for all age groups than in the aforementioned study (La Torre et al., differences ranging from 3 to $6 \mathrm{~cm}$ ). Likewise, P50 depicts higher differences in the group of 3-4-year-olds (from +11 to $+18 \mathrm{~cm}$ for boys and girls) and lower age differences in older groups (i.e. in 5-6-year-olds: from 4 to $8 \mathrm{~cm}$ ). Other studies ${ }^{7,30}$ provided reference values for children from 6 to 10 years old, showing lower variances between 0.5 and 1 year of difference. Moreover, P50 of 6-year-olds from the European IDEFICS study ${ }^{7}$ was higher than in both Spanish pre-schoolers participating in the PREFIT and in Portuguese children. ${ }^{30}$ Once again, this result could be due to the exclusion of overweight and obese children from the data analyses in the IDEFICS study. ${ }^{7}$ In contrast, the Portuguese study and the PREFIT study provide reference values for the whole sample, including all weight status categories. ${ }^{30}$ The standing long jump test is a weight-bearing test where children have to move and lift their body mass. As a result, heavier children usually perform worse than their counterparts with lower body mass. In line with this, Henriksson et al. ${ }^{32}$ confirmed that a better performance on weight-bearing tests in preschool children was associated with a lower fat mass index. The researchers concluded that the more favourable body composition you have, the fitter you are.

Given the differences in the applied methodology, it is not possible to compare our results on motor fitness with any previous study in children and adolescents. In speed-agility (i.e., $4 \times 10 \mathrm{~m}$ shuttle run test), P50 showed the same trend for boys and girls, improving their performance with age, and the range of difference between ages being practically systematic (from 0.2 to nearly $1.8 \mathrm{~s}$ ). The reason for the better performance in older children could be explained by the development produced in motor coordination during the preschool period and childhood. As an example, this improvement was also demonstrated in European children from 6 to 9 years old (P50) who reduced the performance time by one second in $40 \mathrm{~m}$ sprint. ${ }^{7}$ Concerning balance, little is known about the reference standards of this fitness component in pre-schoolers. To the best of our knowledge, only one study ${ }^{33}$ provided static balance norms in children from 4 to 15 years old, yet the data provided for pre-schoolers (4-5 years old) were extracted from a small sample size $(n=25)$ and provided for boys and girls together. In comparison with our data, P50 from the study of Condon and Cremin ${ }^{33}$ was between 2-5 s higher than P50 observed in PREFIT. In our study particularly, P50 of boys and girls followed a similar pattern. Differences between younger and older pre-schoolers were greater in P50 both for boys and girls (approximately $18 \mathrm{~s}$ of difference). It is important to note that, although this test showed low reliability ${ }^{11}$, we decided to provide its reference standard in order to help professionals to detect low levels of this fitness component. Nevertheless, researchers should be cautious when comparing pre-post values on two different occasions or after an intervention programme, due to the low reliability found.

The sex differences observed showed that already from preschool ages differences between boys and girls increased with age. Similar findings were reported by Castro-Piñero et al., ${ }^{34}$ who observed sex differences during the stage from childhood to adolescence. Growth and, particularly, the early maturational status of girls play an important role. Our results are novel because they add the existence of sexual dimorphism in preschool children to the literature and characterise the age-specific pattern of the different development course in both sexes. Nevertheless, further studies are needed in order to corroborate or contrast these findings.

Overall, the present physical fitness reference standards allow other researchers or professionals to classify preschool children in sex- and age-percentiles. Preschool children can also be classified into fitness categories such as very low $(\mathrm{X}<\mathrm{P} 10)$, low $(\mathrm{P} 10 \leq \mathrm{X}<\mathrm{P} 25)$, medium $(\mathrm{P} 25 \leq \mathrm{X}<\mathrm{P} 75)$, high $(\mathrm{P} 75 \leq \mathrm{X}<\mathrm{P} 95)$, and very high $(X \geq P 95)$ and also scaling them from 0 to 10 . In line with this definition, we uploaded an excel-based calculator to the website. With this tool, the researcher or practitioner can copy and paste age, sex, and the result of the fitness test, and the calculator will inform at which percentile that fitness value belongs to. The calculator functions entering either the data of one child, or copying and pasting columns from a data set, for instance of 3000 participants (freely available at http://profith.ugr.es/recursos-prefit). Thereby, professionals (sports practitioners, teachers, health care, trainers, etc.) can identify and help young children classified into the lower categories, implementing strategies to promote physical fitness and physical activity to prevent or reduce future health-related problems. This paper provides valid national specific reference standards for preschool children and thus, our results are valid to compare with Spanish preschool children. However, since no data are available from other countries concerning this population, these reference standards could help and guide professionals in other countries in fitness classification until their own and/or international reference standards are available.

The cross-sectional design of this study does not allow to examine inter- and intra-individual differences, resulting in the need for studies with repeated measurements. The lack of validation studies of the physical fitness applied in preschool children due to the logistic problems inherent to the age of the children is another limitation, yet it must be highlighted that the tests are reliable in pre-schoolers (except for balance). ${ }^{10,11}$ In addition, the difficulty to differentiate between motivation and performance limitations is another study limitation to acknowledge. We also consider the lack of sensitivity analyses for obese children a limitation, since it does not allow to provide specific reference standards for them. Although the analysed sample is not representative for the Spanish population, it is a large sample size that covers cities from north to south and from east to west in Spain. The harmonisation and standardisation of the physical fitness, as well as the use of the GAMLSS as a strong tool to obtain smooth age-dependent reference curves are notable strengths of the study.

\section{Conclusion}

Our study provides age- and sex-specific physical fitness reference standards in Spanish preschool children. This study in children from 3 to 5 years old extends to pre-schoolers the already existing reference standards in older children ${ }^{6,7}$ and adolescents. ${ }^{6,8,35}$ Older children perform better than their younger counterparts. Likewise, sexual dimorphism is detectable already at the age of 3 and increases with age. Overall, boys show better performance than girls in the majority of the applied fitness tests, except for the balance component where girls perform slightly better. These findings will help health, sport, and school professionals to identify preschool children with a 
high/very low fitness level and to examine changes over time, including those obtained due to intervention effects. Further studies should examine and provide reference standards at international level.

\section{Practical implications}

- Reference values are necessary for classifying children, for health screening, and for early prevention as many non-communicable diseases aggravate during growth and development. Therefore, as far as we know, this is the first study providing reference values in preschool children for all fitness components with harmonised measures.

- This study provides sex- and age-specific physical fitness reference standards from a sample of preschool children aged 3-5 years old geographically distributed across 10 cities in Spain $(n=3179)$.

- Boys performed better than girls in the cardiorespiratory fitness, muscular strength, and speed-agility tests, whereas girls performed slightly better in balance tests. Older preschool children performed better in all fitness tests than younger pre-schoolers. Furthermore, sex-related differences in fitness across all preschool ages and percentiles were observed, being greater in older children.

- Tables, Figures, and Supplementary materials help health, sport, and school professionals to identify preschool children with high/ very low fitness levels and to examine changes over time, including those obtained due to intervention effects.

\section{Acknowledgments}

We thank the participation of the preschoolers, parents, and teachers in this study. The PREFIT project takes place thanks to the funding linked to the Ramón y Cajal grant held by FBO (RYC-2011-09011). CC-S is supported by a grant from the Spanish Ministry of Economy and Competitiveness (BES-2014-068829). JRR and FBO are supported by grants from the Spanish Ministry of Science and Innovation (RYC-2010-05957 and RYC-2011-09011, respectively). In addition, this study was further supported by the University of Granada, Plan Propio de Investigación 2016, Excellence actions: Units of Excellence; Unit of Excellence on Exercise and Health (UCEES). Additional funding from the SAMID III network, RETICS, funded by the PN I + D + I 2017-2021 (Spain), ISCIII- Sub-Directorate General for Research Assessment and Promotion, the European Regional Development Fund (ERDF) (Ref. RD16/0022), the EXERNET Research Network on Exercise and Health in Special Populations (DEP2005-00046/ACTI), the University of the Basque Country (GIU14/ 21), and the University of Zaragoza (JIUZ-2014-BIO-08). We are grateful to Ms. Carmen Sainz-Quinn for assistance with the English language. This work is part of a Ph.D. Thesis conducted in the Biomedicine Doctoral Studies of the University of Granada, Spain.

\section{Appendix A. Supplementary data}

Supplementary data associated with this article can be found, in the online version, at https://doi.org/10.1016/j.jsams.2018.09.227.

\section{References}

1. J.R. Ruiz, J. Castro-Pinero, E.G. Artero, et al., Predictive validity of health-related fitness in youth: a systematic review, Br J Sports Med 43 (12) (2009) 909-923.

2. F.B. Ortega, J.R. Ruiz, M.J. Castillo, et al., Physical fitness in childhood and adolescence: a powerful marker of health, Int J Obes (Lond) 32 (1) (2008) 1-11.

3. F.B. Ortega, K. Silventoinen, P. Tynelius, et al., Muscular strength in male adolescents and premature death: cohort study of one million participants, BMJ 345 (2012), e7279.
4. J.J. Lang, K. Belanger, V. Poitras, et al., Systematic review of the relationship between $20 \mathrm{~m}$ shuttle run performance and health indicators among children and youth, J Sci Med Sport 21 (4) (2018) 383-397.

5. B. Martinez-Tellez, G. Sanchez-Delgado, C. Cadenas-Sanchez, et al., Health-related physical fitness is associated with total and central body fat in preschool children aged 3 to 5 years, Pediatr Obes 11 (6) (2016) 468-474.

6. G.R. Tomkinson, J.J. Lang, M.S. Tremblay, et al., International normative $20 \mathrm{~m}$ shuttle run values from 1142026 children and youth representing 50 countries, Br J Sports Med 51 (21) (2017) 1545-1554.

7. P. De Miguel-Etayo, L. Gracia-Marco, F.B. Ortega, et al., Physical fitness reference standards in European children: the IDEFICS study, Int J Obes (Lond) 38 (Suppl. 2) (2014) S57-S66.

8. F.B. Ortega, E.G. Artero, J.R. Ruiz, et al., Physical fitness levels among European adolescents: the HELENA study, Br J Sports Med 45 (1) (2011) 20-29.

9. P.A. Latorre-Roman, F. Garcia-Pinillos, D. Mora-Lopez, Reference values of standing long jump in preschool children: a population-based study, Pediatr Exerc Sci 29 (1) (2017) 116-120.

10. F.B. Ortega, C. Cadenas-Sanchez, G. Sanchez-Delgado, et al., Systematic review and proposal of a field-based physical fitness-test battery in preschool children: the PREFIT battery, Sports Med 45 (4) (2015) 533-555.

11. C. Cadenas-Sanchez, B. Martinez-Tellez, G. Sanchez-Delgado, et al., Assessing physical fitness in preschool children: feasibility, reliability and practical recommendations for the PREFIT battery, J Sci Med Sport 19 (11) (2016) 910-915.

12. C. Cadenas-Sanchez, F. Alcantara-Moral, G. Sanchez-Delgado, et al., Assessment of cardiorespiratory fitness in preschool children: adaptation of the 20 metres shuttle run test, Nutr Hosp 30 (6) (2014) 1333-1343.

13. L.A. Leger, D. Mercier, C. Gadoury, et al., The multistage 20 metre shuttle run test for aerobic fitness, J Sports Sci 6 (2) (1988) 93-101.

14. G. Sanchez-Delgado, C. Cadenas-Sanchez, J. Mora-Gonzalez, et al., Assessment of handgrip strength in preschool children aged 3 to 5 years, J Hand Surg Eur Vol 40 (9) (2015) 966-972.

15. C. Cadenas-Sanchez, G. Sanchez-delgado, B. Martinez-Tellez, et al., Reliability and validity of different models of hand-dynamometers, Am J Occup Ther 70 (4) (2016), 7004300010.

16. R.A. Rigby, D.M. Stasinopoulos, Generalized additive models for location, scale and shape, J R Stat Soc Ser C Appl Stat 54 (2005) 507-554.

17. T. Intemann, H. Pohlabeln, D. Herrman, et al., Estimating age-and height-specific percentile curves for children using GAMLSS in the IDEFICS study, in: A.F. Wilhelm, H.A. Kestler (Eds.), Analysis of large and complex data. Studies in classification, data analysis, and knowledge organization, Springer International Publishing, Heidelberg, 2016, pp. 385-394.

18. R. Gulias-Gonzalez, M. Sanchez-Lopez, A. Olivas-Bravo, et al., Physical fitness in Spanish schoolchildren aged 6-12 years: reference values of the battery EUROFIT and associated cardiovascular risk, J School Health 84 (10) (2014) 625-635.

19. R. Santos, J. Mota, D.A. Santos, et al., Physical fitness percentiles for Portuguese children and adolescents aged 10-18 years, J Sports Sci 32 (16) (2014) $1510-1518$.

20. M.Y. Lam, M.H. Ip, P.K. Lui, M.K. Koong, How teachers can assess kindergarten children's motor performance in Hong Kong, Early Child Dev Care 173 (1) (2003) 109-118.

21. K. Castetbon, T. Andreyeva, Obesity and motor skills among 4 to 6 -year-old children in the United States: nationally-representative surveys, BMC Ped 12 (2012) 28.

22. I. Fjørtoft, Motor fitness in pre-primary school children: the EUROFIT motor fitness test explored on 5-7-year-old children, Pediatr Exerc Sci 12 (4) (2000) 424-436.

23. F.B. Ortega, J.R. Ruiz, I. Labayen, et al., Health inequalities in urban adolescents: role of physical activity, diet, and genetics, Pediatrics 133 (4) (2014) e884-e895.

24. E.G. Artero, V. Espana-Romero, F.B. Ortega, et al., Health-related fitness in adolescents: underweight, and not only overweight, as an influencing factor. The AVENA study, Scand J Med Sci Sports 20 (3) (2010) 418-427.

25. S.C. Dumith, V.V. Ramires, M.A. Souza, et al., Overweight/obesity and physical fitness among children and adolescents, J Phys Act Health 7 (5) (2010) 641-648.

26. F.B. Ortega, B. Tresaco, J.R. Ruiz, et al., Cardiorespiratory fitness and sedentary activities are associated with adiposity in adolescents, Obesity 15 (6) (2007) $1589-1599$.

27. F. Lifshitz, J.P. Hecht, E.F. Bermudez, et al., Body composition analysis by dual-energy X-ray absorptiometry in young preschool children, Eur J Clin Nutr 70 (10) (2016) 1203-1209.

28. P. Allard, M.L. Nault, S. Hinse, et al., Relationship between morphologic somatotypes and standing posture equilibrium, Ann Hum Biol 28 (6) (2001) 624-633.

29. L. Berk, Development through the lifespan, 6th ed., Pearson Higher Education, Boston MA, 2013

30. M.S. Roriz De Oliveira, A. Seabra, D. Freitas, et al., Physical fitness percentile charts for children aged 6-10 from Portugal, J Sports Med Phys Fitness 54 (6) (2014) 780-792. 
31. N. Armstrong, J.R. Welsman, A.M. Nevill, et al., Modeling growth and maturation changes in peak oxygen uptake in 11-13 yr olds, J Appl Physiol 87 (6) (1999) 2230-2236.

32. P. Henriksson, C. Cadenas-Sanchez, M.H. Leppanen, et al., Associations of fat mass and fat-free mass with physical fitness in 4-year-old children: results from the MINISTOP trial, Nutrients 8 (8) (2016) https://doi.org/10.3390/nu8080473.

33. C. Condon, K. Cremin, Static balance norms in children, Physiother Res Int 19 (1) (2014) 1-7.
34. J. Castro-Pinero, J.L. Gonzalez-Montesinos, J. Mora, et al., Percentile values for muscular strength field tests in children aged 6 to 17 years: influence of weight status, J Strength Cond Res 23 (8) (2009) 2295-2310.

35. J.R. Ruiz, I. Cavero-Redondo, F.B. Ortega, et al., Cardiorespiratory fitness cut points to avoid cardiovascular disease risk in children and adolescents; what level of fitness should raise a red flag? A systematic review and meta-analysis, Br J Sports Med (2016) 